\title{
Análisis de la relación entre la autoeficacia interpersonal del profesorado y sus niveles de burnout
}

\author{
Rafael García Ros ${ }^{1}$, María C. Fuentes ${ }^{2}$ y \\ Basilio Fernández ${ }^{3}$
}
${ }^{1}$ Psicología Evolutiva y de la Educación, Universidad de Valencia
${ }^{2}$ Metodología de las Ciencias del Comportamiento, Universidad de Valencia
${ }^{3}$ Conselleria de Educación, Formación y Empleo, Generalitat Valenciana

España

Correspondencia: Rafael García-Ros. Departamento Psicología Evolutiva y de la Educación. Av. Blasco Ibáñez, 21, 46010-Valencia.España. E-mail: Rafael.Garcia@uv.es

(C) Education \& Psychology I+D+i and Ilustre Colegio Oficial de la Psicología de Andalucía Oriental (Spain) 


\section{Resumen}

Introducción. Este trabajo se centra en la evaluación y en el análisis de la capacidad predictiva de la autoeficacia interpersonal del profesorado sobre sus niveles de burnout. Se destaca la utilidad del estudio, dada la ausencia en nuestro contexto de instrumentos de evaluación sobre este constructo psicoeducativo, así como el notable impacto de la percepción de nivel de apoyo social y del mantenimiento de relaciones satisfactorias y de ayuda en el trabajo sobre el estrés docente.

Método. En el estudio participan 103 profesores de distintos niveles del sistema educativo español (55 mujeres, 48 hombres, rango de edad: 24-56), a los que se aplicó una adaptación del Teacher Interpersonal Self-Efficacy Scale -TISES- y la versión española del Maslach Burnout Inventory for Teachers, junto a un cuestionario elaborado ad-hoc que recoge distintas variables sociopersonales del profesorado (sexo, años de experiencia docente y nivel formativo). La validez factorial de la adaptación española del TISES se evalúa a través de un análisis factorial confirmatorio -AFC-, mientras que su validez predictiva e incremental se evalúa a través de diversos análisis de regresión jerárquica múltiple.

Resultados. Se confirma la estructura original del TISES de tres factores oblícuos (percepción de autoeficacia en la gestión del aula, en la obtención de apoyo de compañeros y en la obtenición de apoyo del equipo directivo del centro). Las tres subescalas de autoeficacia muestran niveles de consistencia interna satisfactorios y se relacionan de forma significativa con las dimensiones del burnout -especialmente la percepción de autoeficia en la gestión del aula-, constatando su validez predictiva e incremental sobre los niveles de burnout del profesorado.

Discusión y Conclusión. Se confirma la relación de la percepción de autoeficacia interpersonal del profesorado con sus niveles de burnout, destacando la adecuación y utilidad de la adaptación del TISES. Se discuten los resultados, enfatizando la importancia de promover el desarrollo de la autoeficacia como factor preventivo del burnout, ya sea a través de la mejora de las habilidades instruccionales y de gestión del aula del profesorado, como de la potenciación de sus habilidades de trabajo en equipo y coordinación.

Palabras Clave: Autoeficacia interpersonal, Autoeficacia del profesorado, Burnout docente, Validez factorial, Validez predictiva. 


\section{Teachers' Interpersonal Self-Efficacy: Evaluation and predictive capacity of teacher burnout Abstract}

Introduction. This study analyzed the predictive capacity and incremental validity of teachers' interpersonal self-efficacy on their levels of burnout. First, it presents the validation process of a Spanish adaptation of the Teacher Interpersonal Self-Efficacy Scale -TISES- (Browers \& Tomic, 1999, 2001). Second, the predictive capacity of interpersonal self-efficacy on teacher burnout is analyzed.

Method. The data came to 103 teachers from different levels of the Spanish educational system (55 women, 48 men, age range: 24-56). An adaptation of the TISES, a Spanish version of the Maslach Burnout Inventory for Teachers -MBI-ES-, and a questionnaire developed ad-hoc for various Sociopersonal teacher variables (gender, years of teaching experience and educational level) were applied. The factorial validity of the Spanish adaptation of TISES was assessed with a confirmatory factor analysis -CFA-, while its predictive and incremental validity was assessed through various hierarchical multiple regression analysis.

Results. CFA confirms the TISES three oblique factors original structure: SE in Classroom Management, SE in Eliciting Support from Colleagues, and SE in Eliciting Support from Principals. All the subscales present satisfactory levels of internal consistency and association levels similar to those shown by the original version of the instrument. The three self-efficacy subscales present significant levels of association with the burnout dimensions, and they are shown to be significant predictors of burnout, especially the dimension of SE in Classroom Management. The variables considered in the study explain a high percentage of variance in the different burnout dimensions (42.6\% of Emotional Exhaustion, $45.3 \%$ of Depersonalization and $48.8 \%$ of Personal Accomplishment), showing the incremental validity of interpersonal selfefficacy with regard to the teachers' sociopersonal variables considered.

Discussion and Conclusion. The study confirms the relationship of teachers' interpersonal selfefficacy and burnout, highlighting the appropriateness and usefulness of adapting the TISES. Results are discussed, emphasizing the importance of promoting the development of teachers' self-efficacy as a preventive factor of burnout.

Keywords: Interpersonal Self-Efficacy, Teacher Self-Efficacy, Teacher Burnout, Factorial validity, Predictive validity. 


\section{Introducción}

La autoeficacia docente constituye un importante tópico en la investigación psicoeducativa en las últimas décadas (Duffin, French, \& Patrick, 2012; Woolfolk Hoy, Davis, \& Pape, 2006) dada su estrecha relación con un amplio conjunto de variables instruccionales, con la motivación y con los resultados académicos de los estudiantes en los distintos niveles, modalidades y áreas curriculares del sistema educativo (Caprara, Barbaranelli, Steca, \& Malone, 2006; Faleye, 2008; Hoy y Woolfolk, 1993). Al mismo tiempo, también se relaciona con la eficacia instruccional y con la motivación del profesorado (Woolfolk Hoy, \& Davis, 2006), con su nivel de absentismo y de abandono de la profesión, así como con su satisfacción laboral, nivel de estrés y burnout (p.e., Domènech, 2006, 2009; Evers, Brouwers \& Tomic, 2002; Klassen \& Chiu, 2010; Moè, Pazzaglia, \& Ronconi, 2010; Moriana \& Herruzo, 2004; Skaalvik \& Skaalvik, 2007, 2010; Schwarzer \& Hallum, 2008).

\section{La autoeficacia docente}

La autoeficacia del profesorado presenta notables implicaciones sobre la planificación y desarrollo instruccional, afectando a la definición de objetivos y metas que se plantea el profesorado, a las actividades y métodos de evaluación que aplica (Bandura, 1997), así como al esfuerzo dispuesto a invertir para alcanzarlos. De este modo, un docente con alto sentido de autoeficacia tenderá a pensar que las dificultades de sus estudiantes pueden solucionarse con apoyos, actividades y métodos de evaluación apropiados, con lo que su implicación y persistencia será mayor. Mientras, un docente con reducida autoeficacia tenderá a creer que puede influir en menor grado sobre sus estudiantes, manifestando un nivel de implicación inferior y reduciendo la probabilidad de obtener resultados satisfactorios. Por otro lado, la autoeficacia del profesorado también muestra una estrecha relación con la eficacia colectiva (creencias de los equipos docentes sobre su capacidad para organizar y ejecutar propuestas de acción efectivas), muy ligada a los resultados de los centros educativos debido a que un fuerte sentido de capacidad de grupo establece expectativas de éxito y normas de persistencia y de esfuerzo elevadas (Skaalvik \& Skaalvik, 2007, 2010; Woolfolk Hoy, Davis, \& Pape, 2006).

La investigación también se ha centrado en analizar la relación entre autoeficacia

docente y diferentes variables sociopersonales del profesorado (p.e., sexo, formación 
académica o experiencia laboral), obteniendo resultados no concluyentes. Así, aunque numerosos estudios constatan que las mujeres suelen presentar niveles de autoeficacia superiores a los varones - específicamente en educación infantil y primaria, en educación especial y en la enseñanza universitaria- (Vera, Salanova \& Martín del Río, 2011), otros trabajos señalan lo contrario en distintas situaciones y tareas específicas (p.e., Klassen \& Chiu, 2010). En relación a la formación académica, los resultados señalan que los docentes de educación infantil y primaria con mayor nivel formativo suelen mostar niveles superiores de autoeficacia (Hoy \& Woolfolk, 1993). Por otro lado, aunque distintos trabajos señalan una relación directa entre autoeficacia y experiencia laboral, otros señalan que se produce un incremento significativo en la etapa formativa como docentes y que se ve reducida en el primer año de experiencia profesional, relacionándose con el nivel de apoyo recibido en los centros (Kotaman, 2010; Woolfolk Hoy \& Burke, 2005), mientras que otros estudios señalan que presentan una relación no lineal -decreciendo en la última etapa profesional- (Klassen \& Chiu, 2010).

Pese a que inicialmente la investigación asumió una visión general (p.e., Gibson \& Dembo, 1984), las perspectivas más recientes destacan su naturaleza contextual y situacional, señalando que puede variar entre unas tareas específicas y otras (p.e., un profesor puede percibirse como muy competente para evaluar de forma objetiva el conocimiento de sus estudiantes, pero dudar de su capacidad para desarrollar actividades motivantes en el aula). De este modo, la autoeficacia docente puede definirse como "las creencias de los docentes en su habilidades para organizar y ejecutar las aciones requeridas para lograr el éxito en una tarea específica en un contexto particular" (TschannenMoran, Woolfolk Hoy, \& Hoy, 1998, p. 233). Desde esta perspectiva, resulta lógica la diversidad y amplitud de instrumentos desarrollados (Vera, Salanova, \& Martín-delRío, 2011) dirigidos a su evaluación respecto a distintas habilidades instruccionales (p.e., gestión del aula, Emmer \& Hickman, 1991) o en distintas áreas y modalidades del sistema educativo (p.e., educación especial, Coladarci \& Breton, 1997). En esta línea, Tschannen-Moran and Woolfolk-Hoy (2001) consideran tres dimensiones en su evaluación: (1) estrategias instruccionales, (2) gestión del aula y, (3) implicar a los estudiantes. Y, de forma similar, Cherniss (1993) también destaca la consideración de tres dominios: tareas (habilidades de enseñanza, disciplina y motivación de los estudiantes), interpersonal (habilidades de trabajo con otros, particularmente estudiantes, compañeros y di- 
rectivos del centro) y organización (capacidades para influenciar en los aspectos sociales y políticos en las organizaciones).

\section{Autoeficacia interpersonal del docente}

Este trabajo se centra específicamente en la autoeficacia interpersonal, dada la ausencia de instrumentos dirigidos a su evaluación en castellano, y su especial interés en el contexto educativo al referirse al desarrollo y mantenimiento de relaciones satisfactorias y de ayuda en el trabajo, tanto con estudiantes como con el resto de componentes de los centros educativos (compañeros y equipos directivos). En este sentido, estudios previos en educación secundaria constatan que el nivel de apoyo social percibido por el profesorado presenta un notable impacto sobre su autoeficacia (Brouwers, Evers \& Tomic, 2001) y, especialmente, en los profesores noveles frente a los profesores más experimentados (Tschannen-Moran, \& Woolfolk Hoy, 2007). Adicionalmente, la percepción de falta de apoyo social de los compañeros y del centro destaca entre las principales fuentes de estrés docente, y la percepción de obstáculos laborales relacionados con la misma (p.e., desmotivación e indisciplina de los alumnos o la percepción de falta de apoyo social) juega un papel esencial en el desarrollo del burnout en el profesorado (Llorens, García-Renedo, \& Salanova, 2005).

Desde esta perspectiva, basándose en estudios previos que destacaban la relación entre percepción de falta de apoyo de compañeros y supervisores con el burnout (p.e., Greenglass, Burke \& Konarski, 1997), Brouwers y Tomic (2000, 2001) desarrollan la Teacher Interpersonal Self-Efficacy Scale -TISES- con el objetivo de evaluar la autoeficacia interpersonal del profesorado y comprobar su capacidad predictiva sobre sus niveles de burnout. De forma más concreta, consideran la evaluación de tres dimensiones complementarias: (a) confianza en su capacidad para gestionar eficazmente la conducta de los estudiantes -mantener el orden y la cooperación en clase-, (b) confianza en elicitar el apoyo instrumental y emocional de los compañeros y, (c) confianza en elicitar el soporte del personal directivo del centro. En sus trabajos constatan la validez estructural, adecuación psicométrica y capacidad predictiva de TISES sobre los niveles de burnout del profesorado (Brouwers, Evers y Tomic, 2001), constituyendo en la actualidad uno de los instrumentos de evaluación de la autoeficacia del profesorado más referenciado en estudios posteriores (p.e., Vera, Salanova \& Martín del Río, 2011). 


\section{Objetivo}

Dado el interés de este constructo psicológico, la ausencia de instrumentos disponibles en castellano para su evaluación y su utilidad desde el punto de vista de la evaluación e intervención psicoeducativa en centros escolares, el objetivo de este estudio es analizar la adaptación del TISES a población española, constatando su validez estructural, así como su adecuación psicométrica y validez predictiva sobre los niveles de burnout del profesorado. Además, en el estudio también se consideran distintas variables sociopersonales que han ofrecido resultados controvertidos en cuanto a su relación con la autoeficacia docente (sexo, formación académica y experiencia laboral) y el burnout del profesorado.

\section{Método}

\section{Participantes}

El marco muestral del estudio lo constituyen los centros de Educación Secundaria de la Comunidad Valenciana (España). Se realizó un análisis de la potencia a priori para determinar el tamaño muestral mínimo requerido para detectar con una potencia de $.95(\alpha=.05,1-\beta=.95)$ un tamaño del efecto mediano $\left(f^{2}=.15\right.$, Cohen, 1988). Los resultados indicaron que la muestra debía estar compuesta por un mínimo de 119 participantes (Faul, Erdfelder, Lang \& Buchner, 2009; García, Pascual, Frías, Van Krunckelsven \& Murgui, 2008). Los datos se recogieron de 14 centros seleccionados por muestreo aleatorio simple a partir de todos los centros educativos (Kalton, 1983).

Los participantes en el estudio fueron 103 profesores, 48 hombres (46.6\%) y 55 mujeres $(53.4 \%)$, con edades comprendidas entre 24 y 56 años $(M=38.1$ años, $D T=$ 9.47). Su experiencia profesional oscilaba entre 1 y 33 años $(M=13.47$ años, $D T=$ 9.12). Con respecto a su formación académica, la mayoría de ellos tenían una licenciatura $(86.4 \%)$, mientras que los demás tenían una diplomatura. A pesar de que el tamaño de la muestra fue menor al esperado $(N=103)$, un análisis de la sensibilidad muestral (Faul et al., 2009; García et al., 2008), mostró que se podía detectar un tamaño del efecto ligeramente superior $\left(f^{2}=.17\right)$ con una potencia de .91 . 


\section{Instrumentos y variables}

Tal como se ha mencionado anteriormente, el TISES (Brouwers \& Tomic, 2001, 2002) es un instrumento auto-administrado que está compuesto por 24 ítems que evalúan la confianza de los profesores en sus habilidades interpersonales para (a) manejar el comportamiento de los alumnos en el aula, (b) obtener el apoyo de los colegas, y (c) obtener el apoyo de los equipos directivos de los centros. Las respuestas de los profesores se midieron utilizando una escala de respuesta tipo Likert de 6 puntos, donde 1 corresponde con "completamente en desacuerdo" y 6 con "completamente de acuerdo". De forma más específica, las tres dimensiones de la versión original del TISES son:

- Percepción de autoeficacia en la gestión del aula-GestAula-: Evalúa la confianza del profesorado en gestionar de forma eficaz las situaciones y problemas que pueden surgir en las aulas. Integra 14 ítems -1, 4, 5, 8, 9, 10, 11, 13, 14, 15, 17, 18, 22 y 24 (p.e., "Soy capaz de expresar con claridad a mis alumnos lo que espero de ellos"), con un rango de respuesta entre $14-64(M=3.56, D T=0.61)$, y una consistencia interna de .92 .

- Percepción de autoeficacia en la obtención de apoyo de los compañeros-ApComp-. Evalúa la confianza de los profesores en su capacidad para obtener el apoyo que necesitan en el trabajo por parte de los colegas. Está compuesta por 5 ítems -3, 7, 20, 21 y 23- (p.e., "Cuando es necesario soy capaz de pedir ayuda a mis compañeros"), con un rango de respuesta entre 5-30 $(M=3.77, D T=0.84)$ y una consistencia interna de .91 .

- Percepción de autoeficacia en la obtención de apoyo del equipo directivo-ApDir-. Esta dimensión evalúa la confianza de los profesores en sus habilidades para obtener el apoyo que necesitan en el trabajo por parte de los equipos directivos de los centros. Está compuesta por 5 ítems -2, 6, 12, 16 y 19- (p.e., "Confío en que, cuando resulta necesario, puedo pedir ayuda al equipo directivo de mi centro"), con un rango de respuesta entre 5-30 $(M=3.37, D T=1.13)$ y una consistencia interna de .95 .

El burnout docente fue evaluado a partir de la versión española del Maslach Burnout Inventory para profesores (MBI-ES, Ferrando \& Pérez, 1996), que integra 22 items dirigidos a evaluar los niveles de Agotamiento Emocional, Despersonalización y Realización Profesional del profesorado. Las respuestas de los profesores se midieron 
utilizando una escala tipo Likert de 7 anclajes, donde 1 se corresponde con "nunca" y 7 con "todos los días”. Las tres dimensiones que evalúa el MBI-ES son las siguientes:

- Agotamiento emocional -AE-. Evalúa la disminución y pérdida de recursos emocionales, sensaciones de sobreesfuerzo físico y hastío emocional del profesorado como consecuencia de las contínuas interacciones en su contexto de trabajo. Integra 9 ítems (p.e., "Trabajar con personas me produce demasiada tensión”) con un rango de respuesta entre 9-63. Su consistencia interna es .90 (en este trabajo .87).

- Despersonalización -DP-. Desarrollo de actitudes negativas, de insensibilidad y cinismo hacia los compañeros, padres y alumnos. Consta de 5 ítems (p.e., "Pienso que me he hecho más insensible con la gente") con un rango de respuesta entre 535. Su Su consistencia interna es .79 (en este trabajo .80).

- Realización personal (baja) -RP-. Pérdida de confianza en la consecución de logros en el contexto docente y sentimientos de incompetencia profesional. Integra 8 ítems (p.e., "Me ocupo de manera efectiva de los problemas de mis alumnos") y su rango de respuesta se sitúa entre 8-56. Su consistencia interna es .71 (en este trabajo $.84)$.

Por último, las variables sociopersonales y ocupacionales de los profesores utilizadas en el estudio (sexo, años de experiencia docente y nivel formativo) se obtuvieron a través de un cuestionario desarrollado ad-hoc para este propósito.

\section{Procedimiento}

La versión original del TISES fue traducida al castellano de manera independiente por dos traductores que compararon sus respectivas versiones hasta llegar a un consenso en la traducción que considerada más apropiada. Esta versión preliminar se presentó para su análisis y discusión a un grupo de tres profesores universitarios y dos psicólogos escolares. Después de una cuidadosa consideración, se señaló de manera unánime la necesidad de introducir unos cambios mínimos, obteniendo la versión de la escala que se aplicó a los participantes en el estudio. Distintos colaboradores distribuyeron los cuestionarios entre el profesorado participante en el estudio, encargándose de recogerlos una vez cumplimentados. 


\section{Análisis de datos}

Tras determinar los estadísticos descriptivos de los ítems del TISES se realizó un análisis factorial confirmatorio -AFC- para determinar su validez estructural a través del programa EQS (versión 6.1). Bajo el supuesto de que los tres factores del TISES están relacionados al formar parte del constructo más amplio de autoeficacia interpersonal, se esperaba que un modelo oblicuo de tres factores proporcionara un mejor ajuste a los datos que un modelo unidimensional y un modelo oblicuo de dos dimensiones. En primer lugar, se probó el modelo unidimensional, en el que todos los ítems se incorporaron al constructo latente de percepción de autoeficacia interpersonal (Modelo 0). Posteriormente, el modelo oblicuo de dos dimensiones, asumiendo dos factores relacionados: GestAula y un segundo factor compuesto por los ítems de las otras dos subescalas relativas a apoyo de compañeros y personal directivo de los centros (ApComp y ApDir) (Modelo T1). En tercer lugar, se liberó la restricción de independencia para los errores en los pares de ítems que correlacionaban más intensamente dentro del mismo factor en el modelo oblicuo de dos dimensiones (Modelo Tr1). A continuación se probó el modelo de tres factores correlacionados (Modelo T2) y, por último, basado en este último modelo, se liberaron las covarianzas del error para los pares de ítems más fuertemente correlacionados dentro del mismo factor (Modelo Tr2). Para todos los modelos, se aplicó el procedimiento de máxima verosimilitud con estimación robusta a partir del estadístico Satorra-Bentler Chi-square (Satorra \& Bentler, 2001) y otros índices de ajuste robustos alternativos: el índice de ajuste comparativo (CFI; Bentler, 1990) y la raíz media cuadrática del error de aproximación (RMSEA; Hu \& Bentler, 1999) con su intervalo de confianza del $90 \%$ (CI). Valores $\geq .90$ en CFI muestran un buen ajuste (Medsker, Williams \& Holahan, 1994; Marsh \& Hau, 1996) y los valores en RMSEA $\leq$ .05 son indicativos de un buen ajuste, valores en el rango de .05 - .08 indican un ajuste razonable y valores > .10 indican un ajuste pobre (Browne \& Cudeck, 1992).

Por último, con el objetivo de determinar el nivel de asociación y capacidad predictiva e incremental de las dimensiones del TISES sobre el burnout docente se calculan las correlaciones bivariadas entre las subescalas de autoeficacia y burnout docente, realizando a continuación tres análisis diferentes de regresión lineal jerárquica múltiple considerando como variables dependientes las dimensiones del burnout. 


\section{Resultados}

álisis preliminar de los ítems

Los resultados indicaron que 22 de los 24 ítems mostraban asimetría, y 9 de los ítems mostraban curtosis. La normalidad univariada (DeCarlo, 1997: D'agostinoPearson $K^{2}$ omnibus test; Jarque-Bera LM test) no se confirmó para ningún ítem, estableciendo para las cuatro pruebas un $\alpha=.05$ (DeCarlo, 1997, p. 304; García, Musitu, Riquelme, \& Riquelme, 2011).

\section{Validez Factorial}

Los resultados del AFC mostraron que el modelo oblicuo de tres factores con varianza residual (Modelo $\mathrm{Tr}_{2}$ ) proporciona un mejor ajuste que los modelos alternativos (el modelo unidimensional y el modelo oblicuo de dos dimensiones) (ver Tabla 1). La Figura 1 muestra la representación gráfica del modelo junto con los valores de interrelacion entre las variables.

Tabla 1. Análisis Factoriales Confirmatorios

\begin{tabular}{lccccc}
\hline \multicolumn{1}{c}{ MODELO } & SB- $\chi^{2}$ & $g l$ & SB- $\chi^{2} / g l$ & RMSEA [IC 90\%]* & CFI \\
\hline $\mathrm{Tr}_{2}$. Teórico $+\mathrm{r}_{\text {error } \#}$ & 351.46 & 244 & 1.44 & $.06[.04-.08]$ & .90 \\
$\mathrm{~T}_{2}$. Teórico: 3 Fact. Oblicuos & 434.61 & 249 & 1.74 & $.08[.07-.10]$ & .83 \\
$\mathrm{Tr}_{1}$. Teórico $+\mathrm{r}_{\text {error } \#}$ & 384.88 & 246 & 1.56 & $.07[.06-.09]$ & .87 \\
$\mathrm{~T}_{1}$. Teórico: 2 Fact. Oblicuos & 469.50 & 251 & 1.87 & $.09[.08-.10]$ & .80 \\
U. Unidimensional & 487.23 & 252 & 1.93 & $.10[.08-.11]$ & .78 \\
\hline
\end{tabular}

Nota: $\mathrm{SB}-\chi^{2}=$ Satorra-Bentler chi-square; $g l=$ grados de libertad; RMSEA = raíz media cuadrática del error de aproximación; $\mathrm{CFI}=$ índice de ajuste comparativo.

* IC: intervalo de confianza del $90 \%$ en torno a RMSEA.

\# Modelos $\operatorname{Tr}_{1}$ and $\operatorname{Tr}_{2}$ son los mismos que $\mathrm{T}_{1}$ y $\mathrm{T}_{2}$ respectivamente con la restricción de independencia de los errores en los pares: 8-13, 8-24, 14-22, 20-21 y 2-6. 


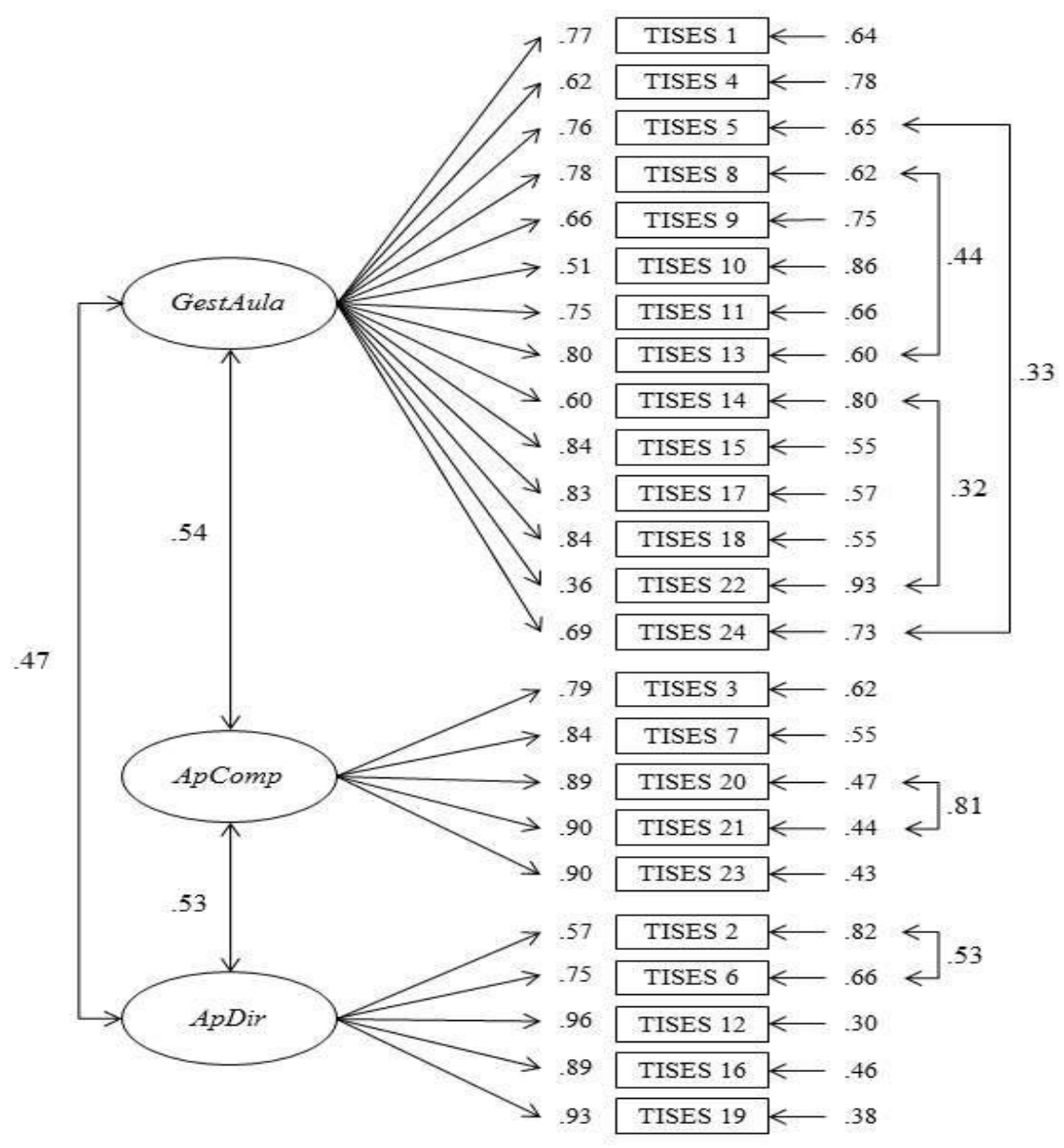

Figura 1. Modelo factorial confirmatorio del TISES

\section{Fiabilidad}

La consistencia interna de las subescalas del TISES se calculó con el alfa de Cronbach. Los resultados mostraron coeficientes de fiabilidad satisfactorios: GestAula, $\alpha=.93$, ApComp,$\alpha=.94$, y ApDir,$\alpha=.92$. Estos valores son similares a los obtenidos en el estudio original (Brouwers et al., 2001).

\section{Autoeficacia Interpersonal y Burnout}

En los distintos análisis de regresión jerárquica el Modelo 1 considera las variables Sexo, Años de experiencia docente y Formación académica como variables expli- 
cativas, mientras que el Modelo 2 también considera las dimensiones de autoeficacia interpersonal.

Se realizó un análisis previo de la relación entre las variables explicativas y las dimensiones del burnout (Tabla 2). En la misma podemos comprobar que el nivel de asociación entre las subescalas del TISES es significativa, obteniendo la correlación más elevada entre ApComp y ApDir $(r=.56, p<.001)$. Adicionalmente, las variables sociopersonales consideradas no presentan una relación significativa con las dimensiones de autoeficacia interpersonal, aunque sí la variable años de experiencia profesional con las dimensiones del burnout. Por último, las dimensiones del TISES y la experiencia docente muestran niveles de asociación significativos con las subescalas del MBI, presentado el valor superior la relación entre GestAula y $R P(r=.64, p<.001)$.

Tabla 2. Nivel de asociación entre las variables consideradas en el estudio

\begin{tabular}{|c|c|c|c|c|c|c|c|c|c|}
\hline Variable & 1 & 2 & 3 & 4 & 5 & 6 & 7 & 8 & 9 \\
\hline 1. Sexo & 1.00 & & & & & & & & \\
\hline 2. Experiencia docente & $-.20^{*}$ & 1.00 & & & & & & & \\
\hline 3. Formación académica & -.08 & .12 & 1.00 & & & & & & \\
\hline 4. Autoef. gestión aula & -.14 & -.00 & .10 & 1.00 & & & & & \\
\hline 5. Autoef. apoyo compañeros & .01 & -.06 & .18 & $.51^{* * *}$ & 1.00 & & & & \\
\hline 6. Autoef. apoyo equipo directiv & -.15 & .12 & .02 & $.50 * * *$ & $.56^{* * * *}$ & 1.00 & & & \\
\hline 7. Agotamiento emocional & -.07 & $.26 * *$ & -.03 & $-.58 * * *$ & $-.37^{* * * *}$ & -.19 & 1.00 & & \\
\hline 8. Despersonalización & -.11 & $.26 * *$ & -.01 & $-.59 * * *$ & $-.44^{* * *}$ & $-.31 * * *$ & $.64 * * *$ & 1.00 & \\
\hline 9. Realización personal & -.01 & $-.22 *$ & -.07 & $.64 * * *$ & $.36^{* * * *}$ & $.43 * * *$ & $-.60 * * *$ & $-.65 * * *$ & 1.00 \\
\hline
\end{tabular}

Los resultados de los análisis de regresión múltiple (Tabla 3) muestran que el Modelo 1 no predice significativamente las puntuaciones en $\operatorname{AE}(F(3,99)=2.5, p=$ $.062)$, y que el Modelo 2 sí que las predice de manera significativa $(F(6,96)=11.9, p<$ .001). Además, el Modelo 2 incrementa significativamente el porcentaje de varianza en AE explicado por el Modelo $1\left(\Delta R^{2}=.16, F(3,96)=19.0, p<.001\right)$. Más específicamente, el Modelo 1 explica un 7.1\% de la varianza del criterio y el Modelo 2 alcanza a explicar un $42.6 \%$ del mismo, con GestAula $(\beta=-.60 ; p<.001)$ y Años de experiencia docente $(\beta=.21 ; p<.05)$ en la ecuación resultante. 
Tabla 3. Síntesis de los análisis de regresion sobre las dimensiones del burnout

\begin{tabular}{|c|c|c|c|}
\hline Variable & $\mathrm{R}^{2}$ & Beta & $T$ \\
\hline Variable dependiente: Agotamiento emocional & .426 & & \\
\hline \multicolumn{4}{|l|}{ Variables independientes } \\
\hline Sexo & & -.10 & -1.2 \\
\hline Experiencia docente & & .21 & $2.6^{*}$ \\
\hline Formación académica & & .02 & 0.2 \\
\hline Autoeficacia gestión aula & & -.60 & $-6.3 * * *$ \\
\hline Autoeficacia apoyo compañeros & & -.15 & -1.5 \\
\hline Autoeficacia apoyo equipo directivo & & .15 & 1.5 \\
\hline Variable dependiente: Despersonalización & .453 & & \\
\hline \multicolumn{4}{|l|}{ Variables independientes } \\
\hline Sexo & & -.13 & -1.6 \\
\hline Experiencia docente & & .22 & $2.8 * *$ \\
\hline Formación académica & & .04 & 0.5 \\
\hline Autoeficacia gestión aula & & -.53 & $-5.7 * * *$ \\
\hline Autoeficacia apoyo compañeros & & -.17 & -1.7 \\
\hline Autoeficacia apoyo equipo directivo & & .01 & 0.1 \\
\hline Dependent variable: Realización personal & .488 & & \\
\hline \multicolumn{4}{|l|}{ Variables independientes } \\
\hline Sexo & & .05 & 0.6 \\
\hline Experiencia docente & & -.22 & $-2.8 * *$ \\
\hline Formación académica & & -.10 & -1.3 \\
\hline Autoeficacia gestión aula & & .57 & $6.4 * * *$ \\
\hline Autoeficacia apoyo compañeros & & -.03 & -.33 \\
\hline Autoeficacia apoyo equipo directivo & & .19 & $2.0^{*}$ \\
\hline
\end{tabular}

$* p<.05, * * p<.01, * * * p<.001$

Adicionalmente, el Modelo 1 tampoco predice de forma significativa las puntuaciones en DP $(F(3,99)=2.6, p=.056)$, mientras que sí lo efectúa el Modelo $2(F(6,96)$ $=13.3, p<.001)$ e incrementa significativamente el porcentaje de varianza explicado por el Modelo $1\left(\Delta R^{2}=.38, F(3,96)=22.2, p<.001\right)$. El Modelo 1 explica el $7.3 \%$ de la varianza de este criterio y el Modelo 2 llega a explicar el 45.3\%, viéndose introducidas en la ecuación de regresión GestAula $(\beta=-.53 ; p<.001)$ y Años de experiencia docente $(\beta=.22 ; p<.01)$.

Por ultimo, el Modelo 1 tampoco predice significativamente las puntuaciones en $\operatorname{RP}(F(3,99)=1.8, p=.14)$, mientras que el Modelo 2 sí lo efectúa $(F(6,96)=15.2, p<$ .001). El Modelo 2 incrementa significativamente el porcentaje de varianza en RP ex- 
plicado por el Modelo $1\left(\Delta R^{2}=.43, F(3,96)=27.1, p<.001\right)$. Por último, destacar que el Modelo 1 explica el 5.4\% de la varianza criterio y el Modelo 2 alcanza a explicar el $48.8 \%$ de la misma, siendo GestAula $(\beta=.57 ; p<.001)$, Años de experiencia docente $(\beta$ $=-.22 ; p<.01)$ y ApDir $(\beta=.19 ; p<.05)$ las variables que se ven introducidas en la ecuación de regresión resultante.

\section{Conclusiones}

Los resultados evidencian la validez factorial y adecuación psicométrica de la adaptación española del TISES. El AFC destaca que el modelo de tres dimensiones oblícuas sobre autoeficacia interpersonal, correspondiente con su estructura original GestAula, ApComp y ApDir-, proporciona un ajuste satisfactorio a los datos, y superior a los modelos alternativos considerados. Los resultados también muestran su adecuación psicométrica, dado que las subescalas obtenidas presentan niveles de consistencia interna satisfactorios y niveles de asociación entre las mismas similares a los destacados en los trabajos originales de validación (Brouwers \& Tomic, 2001; Brouwers et al., 2001). El nivel de asociación superior se evidencia entre ApComp y ApDir, ambos centrados el nivel de confianza del profesorado para elicitar el apoyo instrumental y emocional de compañeros y del personal directivo de los centros en que desarrollan su trabajo.

Por otro lado, tal como preveíamos inicialmente, los niveles de asociación entre las dimensiones de autoeficacia interpersonal y del burnout son significativos, alcanzando valores moderados (nivel de asociación superior de .64 entre GestAula y RP) y en el sentido esperado, de modo que creencias superiores de autoeficacia interpersonal se relacionan con niveles inferiores de burnout. En esta misma línea, Friedman (2003) destaca que tanto la autoeficacia para gestionar el aula (mantenimiento de la disciplina, mantener una comunicación fluida con los estudiantes o ser un instructor efectivo incorporando los comentarios constructivos de compañeros y padres) como la autoeficacia organizacional (percepción de sentirse socialmente aceptado y de influir en los directivos), presentan relaciones significativas inversas con los niveles de burnout del profesorado. Por otro lado, en este trabajo se constata que los niveles de asociación de mayor magnitud con el burnout del profesorado se alcanzan con GestAula (rango entre .58 y 
.64), lo cual apoya la idea que la confianza del profesorado en su capacidad para gestionar el comportamiento y las problemáticas que puedan generar los estudiantes en el aula, así como mantener un clima de trabajo adecuado y la cooperación en clase, es la dimensión de la autoeficacia interpersonal que actúa como principal factor protector del burnout. Estos resultados también coinciden con los destacados en los estudios originales de validación del TISES y con estudios longitudinales efectuados con profesorado de educación secundaria (Brouwers \& Tomic, 2000; Brouwers et al., 2001).

En el trabajo también se ha constatado la inexistencia de relaciones significativas entre las variables sociopersonales del profesorado consideradas y las dimensiones de autoeficacia interpersonal, aunque los años de experiencia profesional sí muestran una relación positiva con el burnout. Los análisis de regresión evidencian la validez incremental de las dimensiones de autoeficacia sobre las variables sociopersonales del profesorado para predecir sus niveles de burnout, llegando a explicar conjuntamente un $43 \%$ de varianza de $A E$, un $45 \%$ de $D P$, y un $49 \%$ de $R P$. De forma más específica, en las ecuaciones de regresión resultantes dos escalas de autoeficacia contribuyen de forma significativa a predecir el burnout, GestAula -en todas las dimensiones de burnout- y ApDir -exclusivamente para RP--. De forma similar, Friedman (2003) concluye que los principales predictores del nivel burnout del profesorado son (a) su autoeficacia para promover el que los estudiantes expresen sus sentimientos y opiniones en el aula de cara a responder a sus necesidades cognitivas y emocionales, y (b) su percepción de sentirse socialmente aceptado y poder influir sobre las opiniones y decisiones de los equipos directivos. Sin embargo, las ecuaciones de regresión resultantes en su estudio presentan una capacidad explicativa sobre el burnout sensiblemente inferiores a las obtenidas en este trabajo (20\% de varianza en $D P, 5 \%$ en $A E$ y $8 \%$ en $R P)$.

En definitiva, este estudio confirma la importancia y relación de la percepción de autoeficacia interpersonal con los niveles de burnout del profesorado, destacando la validez y adecuación psicométrica de la adaptación a lengua castellana del TISES, que constituye una herramienta de especial interés en el contexto educativo dado que evalúa la percepción del mantenimiento de relaciones satisfactorias y de ayuda en el trabajo tanto con estudiantes como con el resto de componentes de los centros educativos (compañeros y directivos)-, así como su elevada capacidad predictiva sobre los niveles de burnout del profesorado. Estudios posteriores deben seguir analizando la relación 
entre variables sociopersonales del profesorado, autoeficacia interpersonal incorporando las relaciones con los padres de los estudiantes- y niveles de burnout, siendo especialmente importante explorar la relación causal entre estas dos últimas, dadas las notables implicaciones para la comprensión e intervención eficaz en este ámbito. En esta línea, tal como señalan Skaalvik \& Skaalvik (2010), probablemente la relación entre autoeficacia y burnout sea recíproca, dado que una reducida autoeficacia puede incrementar los niveles de burnout, pero también elevados niveles de burnout pueden provocar que el profesorado obtenga resultados inferiores en su actividad docente que, a su vez, pueda afectar a su percepción de autoeficacia. Por otro lado, la investigación también debe enfatizar el análisis de las intervenciones que promueven el desarrollo de la autoeficacia como factor preventivo del burnout del profesorado, ya sea a través de la mejora de sus habilidades instruccionales y de gestión del aula, como de la potenciación de sus habilidades de trabajo en equipo y coordinación con compañeros y directivos de los centros.

\section{Referencias}

Bandura, A. (1997). Self-efficacy: The exercice of control. New York: W.H. Freeman. Bentler, P. M. (1990). Comparative fit indexes in structural models. Psychological Bulletin, 107, 238-246. Doi:10.1037/0033-2909.107.2.238

Brouwers, A., \& Tomic, W. (1999). Teacher burnout, perceived self-efficacy in classroom management, and student disruptive behavior in secondary education. Curriculum and Teaching, 14(2), 7-26.

Brouwers, A., \& Tomic, W. (2000). A longitudinal study of teacher burnout and perceived self-efficacy in classroom management. Teaching and Teacher Education, 16, 239-253.

Brouwers, A., \& Tomic, W. (2001). The factorial validity scores on the Teacher Interpersonal Self-efficacy scores. Educational and Psychological Measurement, 61 (3), 433-445.

Brouwers, A., Evers, W.J.G., \& Tomic, W. (2001). Self-efficacy in eliciting social support and burnout among secondary-school teachers. Journal or Applied Social Psychology, 31 (7), 1474-1491.

Browne, M. W. \& Cudeck, R. (1992). Alternative ways of assessing model fit. Sociological Methods and Research, 21, 230-258. Doi: $10.1177 / 0049124192021002005$. 
Caprara, G.V., Barbaranelli, C., Steca, P., \& Malone, P.S. (2006). Teachers selfefficacy as determinants of job satisfaction and students' academic achievement: A study at the school level. Journal of School Psychology, 44, 473-490.

Cherniss, C. (1993). Role of professional self-efficacy in the etiology and amelioration of burnout. En. W.B. Schaufeli; C. Maslach, \& T. Marek (Eds.) (1993). Professional burnout. Recents developments in theory and research. Washington D.C.: Hemisphere.

Cheung, W. M., \& Cheng, Y. C. (1997). A multi-level analysis of teacher's self belief and behavior, and student's educational outcomes. Paper presented at the Annual Meeting of American Educational Research Association: Talking Together in Educational Research \& Practice, Chicago, IL.

Cohen, J. (1988). Statistical power analysis for the behavioral sciences (2nd ed.). Hillsdale, N.J.: Erlbaum.

Coladarci, T., \& Breton, W. (1997). Teacher efficacy, supervisión, and the special education resource-room teacher. Journal of Educational Research, 90, 230-239.

DeCarlo, L. T. (1997). On the meaning and use of kurtosis. Psychological Methods, 2, 292-307. Doi:10.1037/1082-989X.2.3.292

Domenech, F. (2006). Stressors, Self-Efficacy, Coping Resources, and Burnout among Secondary School Teachers in Spain. Educational Psychology: An International Journal of Experimental Educational Psychology, 26 (4), 519-529.

Domenech, F. (2009). Self-efficacy, school resources, job stressors and burnout among Spanish primary and secondary school teachers: a structural equation approach. Educational Psychology: An International Journal of Experimental Educational Psychology, 29(1), 45-68.

Duffin, L.C., French, B.F., \& Patrick, H. (2012). The Teachers' Sense of Efficacy Scale: Confirming the factor structure with beginning pre-service teachers. Teaching and Teacher Education, 28, 827-834.

Emmer, E., \& Hickman, J. (1991). Teacher efficacy in classroom management. Educational and Psychological Measurement, 51, 755-765.

Evers, W.J.G.; Brouwers, A. \& Tomic, W. (2002). Burnout and self-efficacy: A study of teachers' beliefs when implementing an innovative educational system in the Netherlands. British Journal of Educational Psychology, 72, 227-243.

Faul, F., Erdfelder, E., Buchner, A., \& Lang, A. G. (2009). Statistical power analyses using G*Power 3.1: Tests for correlation and regression analysess. Behavior Research Methods, 41(4), 1149-1160. Doi:10.3758/BRM.41.4.1149.

Faleye, B. A. (2008). Análisis de fiabilidad y de factores de una escala de eficacia docente para profesores de educación secundaria de Nigeria. Electronic journal of research in educational psychology, 6(16), 823-846. 
Friedman, I.A. (2003). Self-efficacy and burnout in teaching: the importance of interpersonal-relations efficacy. Social Psychology of Education, 6, 191-215.

García, J. F., Pascual, J., Frías, M. D., Van Krunckelsven, D., \& Murgui, S. (2008). Design and power analysis: $\mathrm{n}$ and confidence intervals of means. Psicothema, 20, 933-938.

Gibson, S., \& Dembo, M. (1984). Teacher efficacy. A construct validation. Journal of Educational Psychology, 76, 569-582.

Goddard, R. D.; Hoy, W. K. \& Woolfolk Hoy, A. (2004). Collective efficacy beliefs: Theoretical developments, empirical evidence, and future directions. Educational Researcher, 33(3), 3-13.

Greenglass, E. R.; Burke, R. J. \& Konarski, R. (1997). The impact of social support on the development of burnout in teachers: examination of a model. Work \& Stress, 11, 267-278.

Hastings, R.P., \& Bham, M.S. (2003). The relationship between student behaviour pattern and teacher burnout. School Psychology International, 24(1), 115-127.

Hoy, W. K., \& Woolfolk, A. E. (1993). Teacher's sense of efficacy and the organizational health of schools. The Elementary School Journal, 93, 356-372.

Hu, L. \& Bentler, P. M. (1999). Cutoff criteria for fit indexes in covariance structure analysis: Conventional criteria versus new alternatives. Structural Equation Modeling: A Multidisciplinary Journal, 6, 1-55. Doi: $10.1080 / 10705519909540118$.

Kalton, G. (1983). Introduction to survey sampling. Beverly Hills, CA: Sage.

Klassen, R. M., \& Chiu, M. M. (2010). Effects on teachers' self-efficacy and job satisfaction: Teacher gender, years of experience, and job stress. Journal of Educational Psychology, 102, 741-756.

Kotaman, H. (2010). Turkish early childhood educators' sense of teacher efficacy. Electronic Journal of Research in Educational Psychology, 8(2), 603-616.

Llorens, S.; García-Renedo, M., \& Salanova, M. (2005). Burnout como consecuencia de una crisis de eficacia: Un estudio longitudinal en profesores de secundaria. $R e-$ vista de Psicología del Trabajo y de las Organizaciones, 21(1-2), 55-70.

Marsh, H. W. \& Hau, K.-T. (1996). Assessing goodness of fit: Is parsimony always desirable? Journal of Experimental Education, 64, 364-390.

Medsker, G. J., Williams, L. J., \& Holahan, P. J. (1994). A review of current practices for evaluating causal-models in organizational-behavior and human-resources management research. Journal of Management, 20, 439-464. Doi: 10.1016/0149-2063(94)90022-1. 
Moè, A., Pazzaglia, F. \& Ronconi, L. (2010). When being able is not enough. The combined value of positive affect and self-efficacy for job satisfaction in teaching. Teaching and Teacher Education, 26, 1145-1153.

Moriana. J.A., \& Herruzo, J. (2004). Estrés y burnout en profesores. International Journal of Clinical and Health Psychology, 4(3), 597-621.

Satorra, A. \& Bentler, P. M. (2001). A scaled difference chi-square test statistic for moment structure analysis. Psychometrika, 66, 507-514. Doi:10.1007/BF02296192

Schwarzer, R,. \& Hallum, S. (2008). Perceived teacher self-efficacy as a predictor of job stress and burnout: mediation analyses. Applied Psychology, 57, 152-171.

Skaalvik, E.M., \& Skaalvik, S. (2007). Dimensions of teacher self-efficacy and relations with strain factors, perceived collective teacher efficacy, and teacher burnout. Journal of Educational Psychology, 99(3), 611-625.

Skaalvik, E.M., \& Skaalvik, S. (2010). Teacher self-efficacy and teacher burnout: A study of relations. Teacher and Teaching Education, 26, 1059-1069.

Tschannen-Moran, M., \& Woolfolk Hoy, A. (2001). Teacher efficacy: Capturing and elusive construct. Teacher and Teaching Education, 17, 783-805.

Tschannen-Moran, M., \& Woolfolk Hoy, A. (2007). The differential antecedents of self-efficacy beliefs of novice and experienced teachers. Teacher and Teaching Education, 23, 944-956.

Tschannen-Moran, M., Woolfolk Hoy, A. \& Hoy, W. K. (1998). Teacher efficacy: Its meaning and measure. Review of Educational Research, 68, 202-248.

Vera, M., Salanova, M., \& Martín-del-Río, B. (2011). Self-efficacy among university faculty: how to develop an adjusted scale. Anales de Psicología, 27(3), 800-807.

Woolfolk Hoy, A. \& Burke, R. (2005). Changes in teacher efficacy during the early years of teaching: A comparison of four measures. Teacher and Teaching Education, 21, 343-356.

Woolfolk Hoy, A., Davis, H. \& Pape, S. J. (2006). Teacher knowledge and beliefs. In P. A. Alexander \& P. H. Wine: Handbook of Educational Psychology, 715-737. NJ. LEA. 